\title{
Electrochemical glucose biosensor with the characterization of surface morphology and content of glucose oxidase- glutaraldehyde-cysteine layers on gold electrode
}

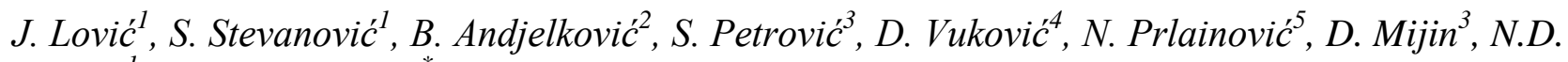 \\ Nikolic ${ }^{1}$, M. Avramov Ivic ${ }^{*}$ \\ ${ }^{1}$ ICTM - Institute of Electrochemistry, University of Belgrade, Njegoševa 12, 11000, Belgrade, Serbia \\ ${ }^{2}$ Faculty of Chemistry, University of Belgrade, Studentski trg 12-16, 11000 Belgrade, Serbia \\ ${ }^{3}$ Faculty of Technology and Metallurgy, University of Belgrade, Karnegijeva 4, 11000 Belgrade, \\ Serbia \\ ${ }^{4}$ Faculty of Medicine, University of Belgrade, Dr Subotića 8, 11000 Belgrade, Serbia \\ ${ }^{5}$ Innovation Center of Faculty of Technology and Metallurgy, University of Belgrade, Karnegijeva 4, \\ 11000 Belgrade, Serbia \\ *E-mail: milka@tmf.bg.ac.rs
}

Received: 3 July 2018 / Accepted: 25 July 2018 / Published: 5 November 2018

Glucose biosensor containing cysteine (Cys), glutaraldehyde (GA) and glucose oxidase (GOx) onto gold electrode is constructed and already electrochemically tested. Now, the electrochemical behavior of biosensor in human serum is further investigated and supported by morphological characterization of layers for the first time. The morphology and microstructure of layers was examined by Fourier transformed infra red spectroscopy (FTIR), atomic force (AFM) and optical microscopy (OM). The electrochemical indication that the Cys-GA-GOx film on Au surface is not compact and that there were some bare regions which remain catalytically active is supported by AFM and OM results. The construction and the nature of bonding of Au-Cys-GA-GOx biosensor layers is confirmed by the FTIR study.

Keywords: Biosensor, Electrochemical glucose sensing, Microstructural characterization, FTIR, AFM, OM microstructural characterization.

\section{INTRODUCTION}

The number of people with diabetes is growing rapidly $[1,2]$. Improved glycemic control reduced the risk of progression of complications related to diabetes mellitus. The limitations of old concepts of measuring glycemia require new methods to assess bodily biochemistry as fast and easier as possible, so to diagnose diabetic eye disease in the early stage. The development of an efficient 
biosensor for monitoring blood glucose levels would be great tool combating diabetes mellitus [3-8].

There is a need to develop an electrochemical glucose biosensor by immobilizing enzyme on an electrode surface. Gold is usually used for immobilization of enzymes [9-11] and the first modification step involve compounds with thiol (-SH) group [12-15], or cysteine as cheaper and more stable. Glutaraldehyde (GA) is a well known powerful crosslinker with reactive aldehyde groups on both ends and one of the most widely used reagents for the activation of aminated surface [16]. GOx is a first choice as a biosensing model molecule in many studies because it is stable, relatively low cost compound [17, 18], and frequently used in clinical praxis [19-21].

Numerous biosensors were already constructed and tested [22-27]. Biosensor containing Cys, GA and GOx on gold electrode was constructed and it is preliminary electrochemically investigated and compared with similar sensors found in available literature data [28]. The glucose oxidaseglutaraldehyde-cysteine modified gold electrode as a biosensor exhibits excellent sensitivity, selectivity and stability for glucose monitoring. The important advantage of the investigated system enables proper glucose detection in biological systems because of the lack of interferences with $\mathrm{O}_{2}$ or $\mathrm{H}_{2} \mathrm{O}_{2}$ [28].

In this work we continue development of our biosensor in human serum with the physical insight in its layers obtained by successive attachment of Cys, GA and GOx onto gold electrode. The electrochemical performances of biosensor are presented showing the effective potential area for glucose sensing in human serum. The surface morphology of biosensor and structure of layers of modified gold electrode was analyzed and characterized by FTIR, AFM and OM techniques and connected with the electrochemical ability.

\section{MATERIALS AND METHODS}

\subsection{Chemicals}

Glucose oxidase from Aspergillus niger (EC 1.1.3.4), Type VII, $149800 \mathrm{U}$ g-1 ( $150 \mathrm{U}$ mg-1 solid), L-cysteine and D-(+)-glucose were purchased from Sigma Aldrich. Glutaraldehyde was purchased from Acros Organics, and salts for the phosphate buffer from Merck Alkaloid. Milipore Waters Milli-Q purification unit was used in order to obtain deionized water.

The modification of Au electrode with cysteine (Au-Cys) was performed as it was described previously [28].

\subsection{Electrochemical experiment}

For cyclic voltammetry (CV), PGZ 402 Volta Lab (Radiometer Analytical, Lyon, France) was used. The three electrode electrochemical cell and the preparation of polycrystalline gold electrode (surface area $0.5 \mathrm{~cm}^{2}$ ) were described in detail previously [29]. A gold wire was used as the counter electrode and a saturated calomel electrode (SCE) as the reference electrode. All potentials are given vs. SCE. The electrolytes were deoxygenated by purging with nitrogen. 
The human serum was collected and clinically prepared from twenty healthy volunteers and spiked with glucose using procedure described previously [30].

\subsection{Surface characterization}

IR spectra were recorded on a Thermo Scientific Nicolet 6700 FT-IR spectrometer, using the attenuated total reflectance (ATR) technique from the Smart accessory with diamond crystal (Smart Orbit, Thermo Scientific, Madison, WI, USA). Spectral data were collected in the mid-IR range (1800$600 \mathrm{~cm}^{-1}$ ) with 64 scans and $2 \mathrm{~cm}^{-1}$ resolution. A background spectrum (32 scans) was recorded before every sample spectrum. IR spectra were baseline corrected and smoothed using OMNIC software (version 7.0, Thermo Scientific, USA), and exported to SPC files [31].

Atomic force microscope (AFM) was employed to observe the gold surface after each modification step. NanoScope III A (Veeco Digital Instruments, USA) microscope was used. Tapping mode AFM images were recorded at the room temperature using etched silicon cantilevers with force constant $60 \mathrm{~N} / \mathrm{m}$. Image analysis was done by Nanoscope image processing software. The surface characterization was also performed using optical microscope (Olympus CX41) connected to the computer.

\section{RESULTS AND DISCUSSION}

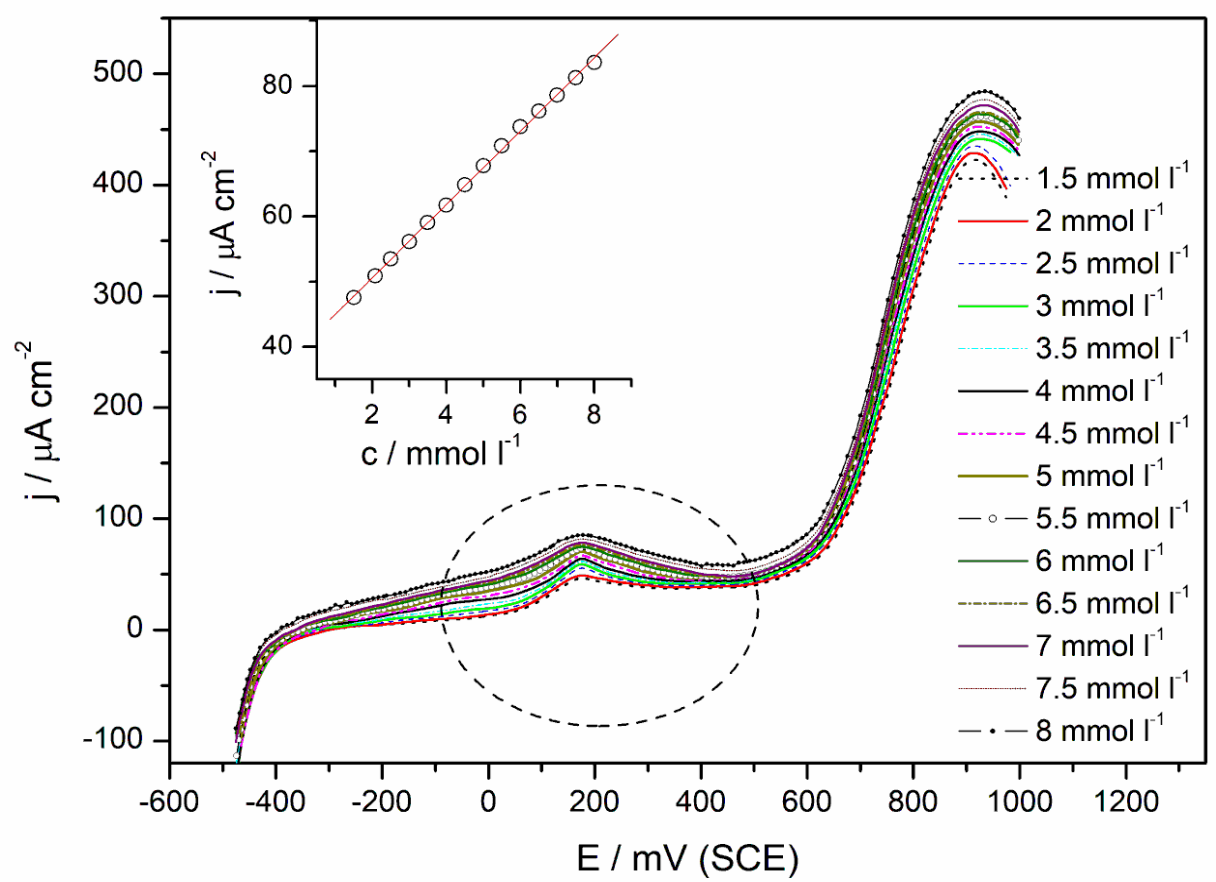

Figure 1. CVs obtained on Au-Cys-GA-GOx electrode using phosphate buffer $(0.1 \mathrm{M}, \mathrm{pH} 7.0)$ for different glucose concentration spiked with human serum at scan rate of $100 \mathrm{mVs}^{-1}$. Inset: $\mathrm{j}$ vs. E from $-200 \mathrm{mV}$ to $400 \mathrm{mV}$.

The ability of the presented biosensor for the glucose measuring was tested by CV for the 
different glucose concentrations spiked with human serum as is presented in Fig. 1. The serum samples obtained from 20 healthy volunteers, spiked with the glucose concentrations (clinical values for healthy people $[30,32]$ gave the same electrochemical response.

In the presented range of the glucose concentrations there is a linear relationship between current vs. glucose from 1.5 to $8 \mathrm{mmol}^{-1}$, as is presented in inset in Fig. 1. The linear current/glucose concentrations dependency is observed in the area of the potential from $-200 \mathrm{mV}$ to $400 \mathrm{mV}$.

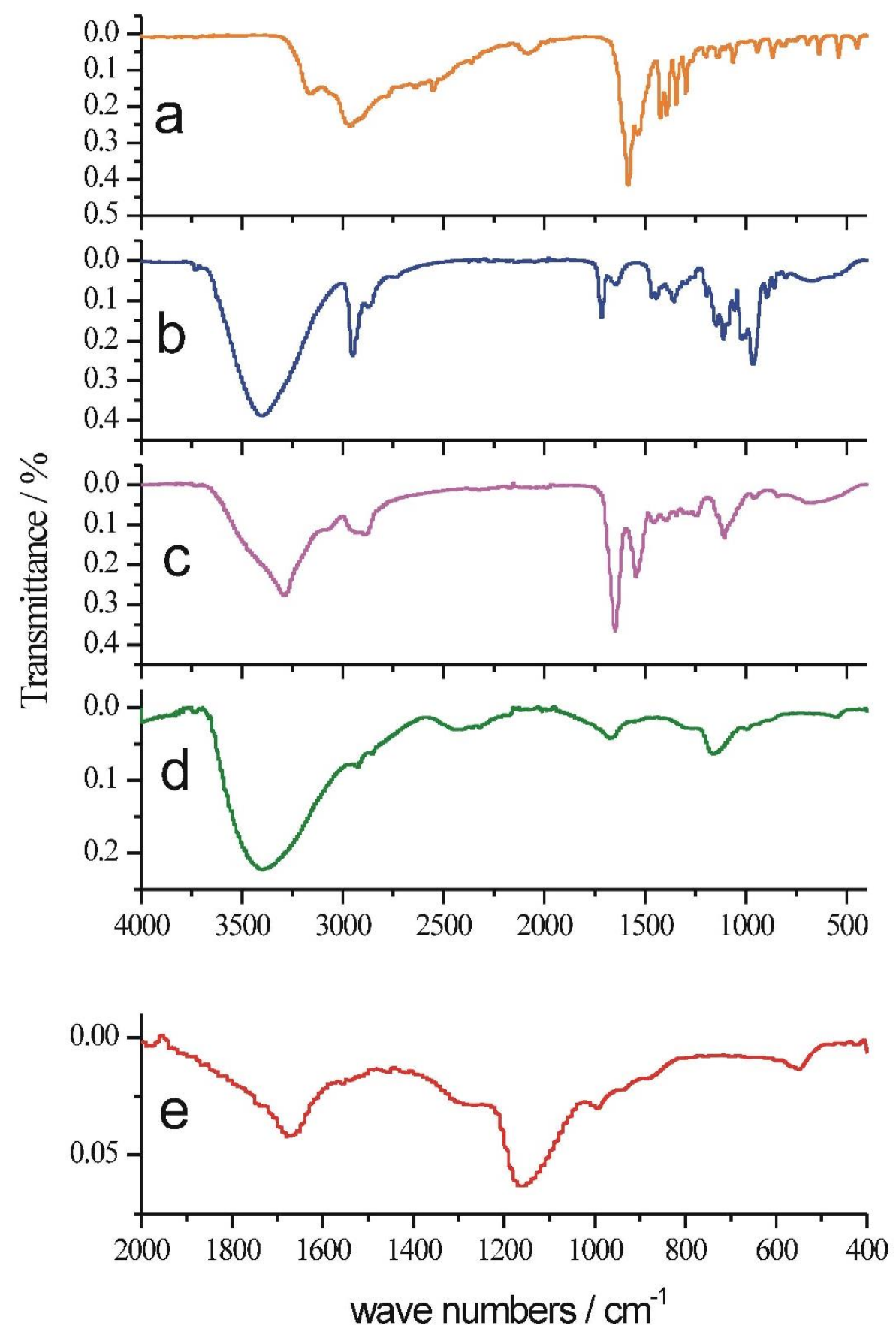

Figure 2. FTIR spectra of: a) cysteine, b) glutaraldehyde, c) glucose oxidase, d) Au-Cys-GA-GOx

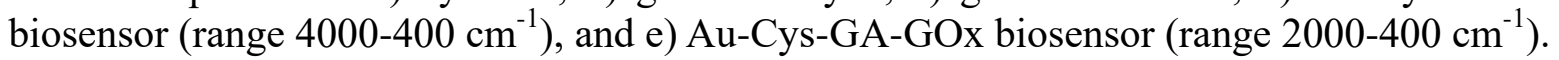


In this way the effective potential area for glucose sensing in human serum is established and it is explained as follows. It is well known that $\mathrm{OH}^{-}$enables glucose oxidation and the $\mathrm{AuOH}$ formation on the gold surface in the potential range -0.1 to $0.3 \mathrm{~V}$ occurs [33]. This indicates that the Cys-GA$\mathrm{GOx}$ film on $\mathrm{Au}$ surface is not compact and that there were some bare regions which remain catalytically active. It is supported by AFM and OM results and will be discussed in detail. It is important to notice that glucose oxidation in the whole range of the potential presented in Fig1 proceeds with 50\% lower anodic currents on bare gold electrode than on the sensor surface [28].

In order to improve further the physical insight in the construction and the nature of bonding of Au-Cys-GA-GOx biosensor layers, the FTIR study is performed and presented in Fig. 2.

Spectra of Cys, GA and GOx (Fig. 2. a, b, c) exhibit all the characteristic bands described in the literature [34-36]. Au electrode modification is elucidated and confirmed by observing the part of the spectrum between 1800 and $500 \mathrm{~cm}^{-1}$ (Fig. 2e). The absence of band at $1715 \mathrm{~cm}^{-1}$, that in the spectrum of $\mathrm{GA}$ originates from $-\mathrm{C}=\mathrm{O}$ group, indicates that reaction with enzyme amino groups occurred. The most prominent bands in the spectrum of biosensor at 1675 and $1160 \mathrm{~cm}^{-1}$ originates from the amide I band and enzyme carbohydrate moiety and undoubtedly confirms the attachment of glucose oxidase. In addition, the band at $1675 \mathrm{~cm}^{-1}$ is broader in comparison to amide I band in the spectrum of free enzyme what can be explained by overlapping with absorption of imino group formed by the reaction of glutaraldehyde carbonyl group and enzyme amino group [37].

In order to obtain better insight in characteristics of this biosensor, it was necessary to make analysis of all elements or layers of which the biosensor is constructed. The surface characterization of the initial surface area $(\mathrm{Au})$, as well as the surface areas after the added Cys, GA and GOx are done by the AFM technique and the change in the surface morphology after each subsequent modification step are presented in Fig. 3.

AFM images of pure Au surface area (Fig. 3a) and those obtained with the addition of cysteine (Au-Cys; Fig. $3 b$ and c). The AFM analysis showed that the RMS roughness of the surface area of $\mathrm{Au}$ with the adsorbed cysteine (Au-Cys; Fig. 3b) compared with the same surface area of pure Au was larger for about $30 \%$ (Fig. 3a). Also, it can be seen from Fig. 3c that molecules of cysteine do not cover the surface area of Au completely. This is in a complete accordance with the effective potential area presented in inset (Fig. 1) and with the catalytic influence of pure gold electrode uncovered by biosensor layers.

The attachment of GA was confirmed by the AFM technique as shown in Fig. $3 \mathrm{~d}$. The line section analysis of the part of surface area shown in Fig. $3 \mathrm{~d}$ is given in Fig. 3e, from which can be seen that the size of GA agglomerates was around $300 \mathrm{~nm}$. It is important to note that they are uniform over the surface, in a size as well as in a distribution.

The immobilization of GOx on GA activated surface proceed through a two step mechanism with the first being adsorption and the second covalent bonding [38]. By choosing Cys and GA as linkers the adsorption was reduced to a minimum as it was previously confirmed [39]. It can be seen from Fig. $3 \mathrm{f}$ that the surface area is not completely covered with the enzyme. Small enzyme aggregates $(10-20 \mathrm{~nm})$ are properly grouped over the previously introduced GA. 
a

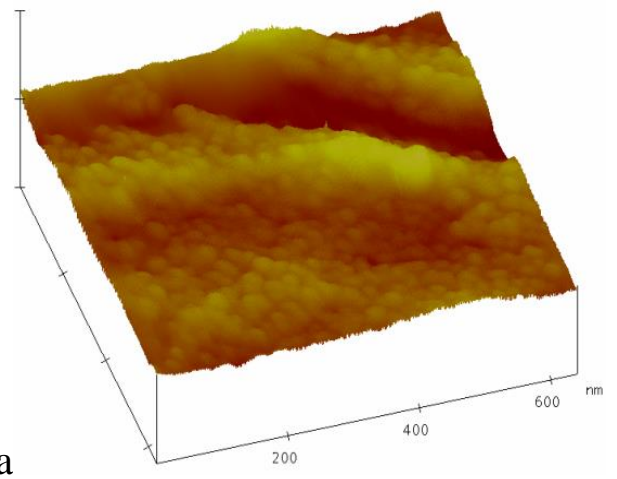

c
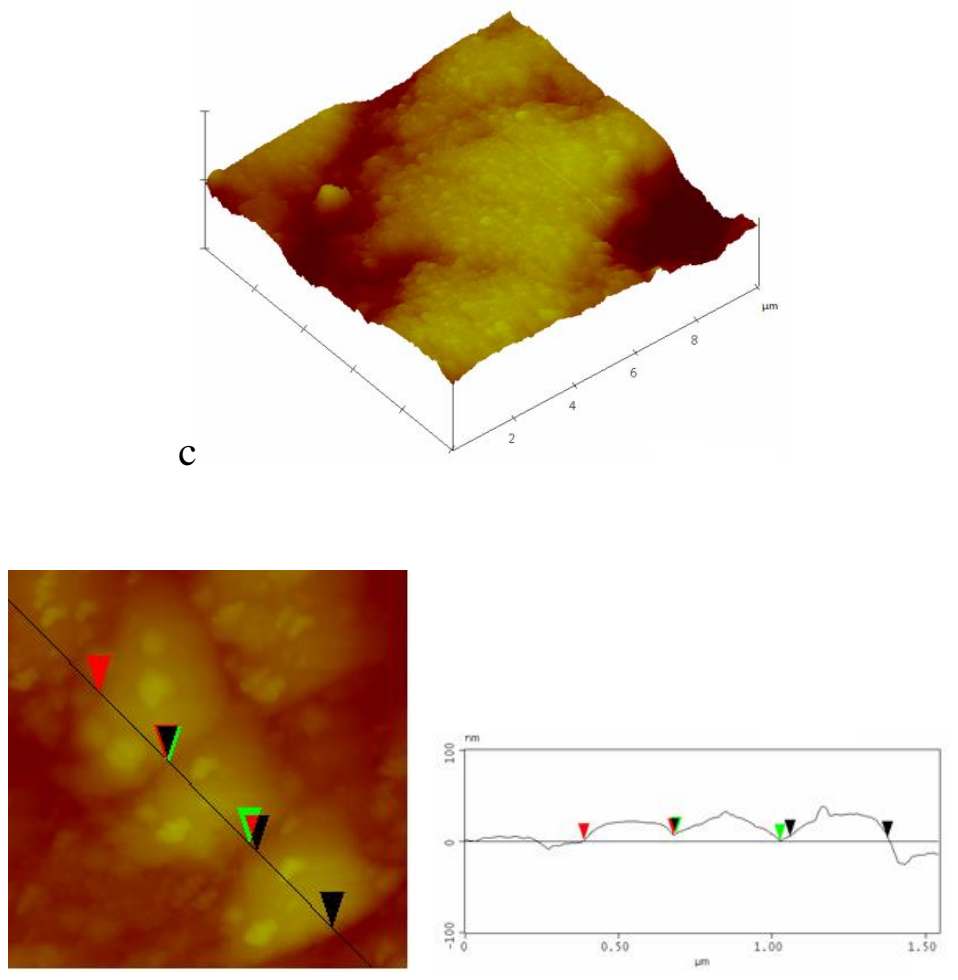

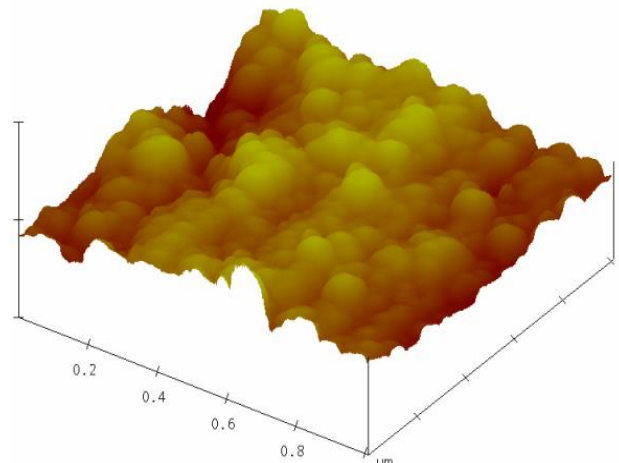

$\mathrm{b}$
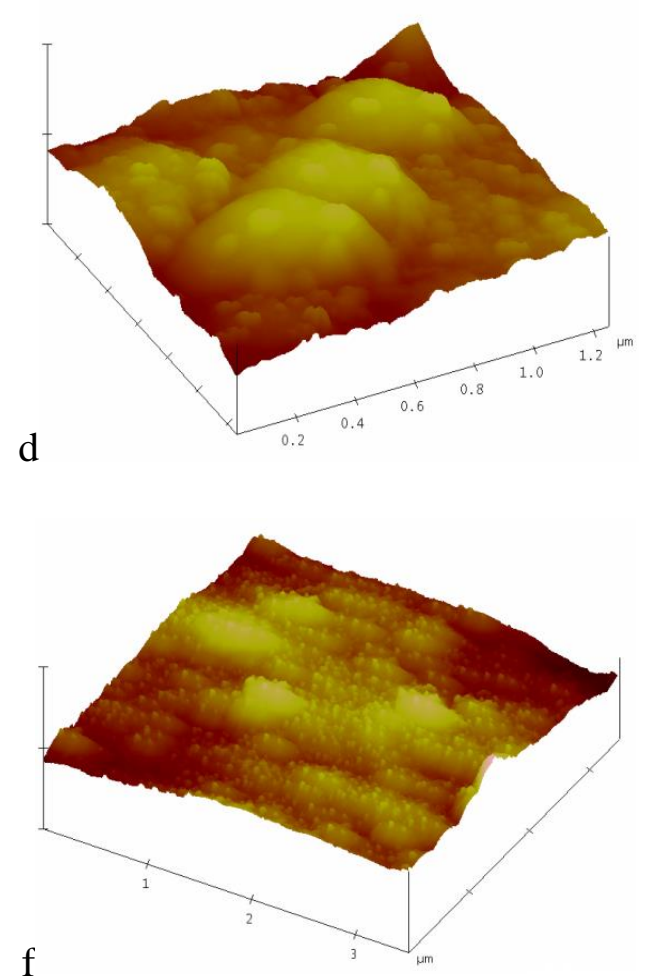

$\mathrm{e}$

Figure 3. a) $\mathrm{Au}(0.65 \times 0.65 \times 0.1 \mu \mathrm{m})$; (RMS: $5.875 \mathrm{~nm})$, b) Au-Cys $(1 \times 1 \times 0.15 \mu \mathrm{m})$; (RMS: 8.704 $\mathrm{nm})$, c) Au-Cys $(10 \times 10 \times 1.5 \mu \mathrm{m})$, d) Au-Cys-GA $(1.25 \times 1.25 \times 0.2 \mu \mathrm{m})$, e) top view and line section analysis of the part of surface area under d), and f) Au-Cys-Ga-GOx $(3 \times 3 \times 0.2 \mu \mathrm{m})$.

Due to specific crystallization of serum on the biosensor surface area, the further characterization of the biosensor was done by the OM technique. Figure 4 shows the surface area of the biosensor (Fig. 4a and b), as well as the surface areas of the biosensor with the added glucose (Fig. $4 \mathrm{c}$ and d), with the added glucose and serum (Fig. 4e, f and g), and with the added serum only (Fig. 4h and i). 


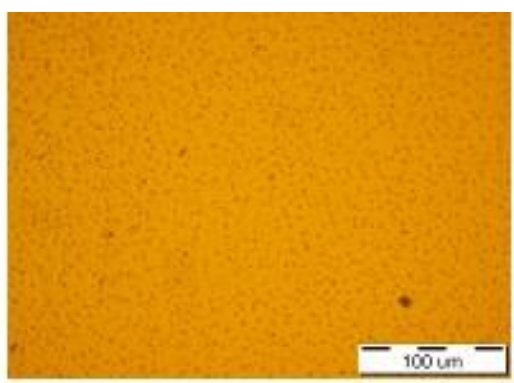

a)

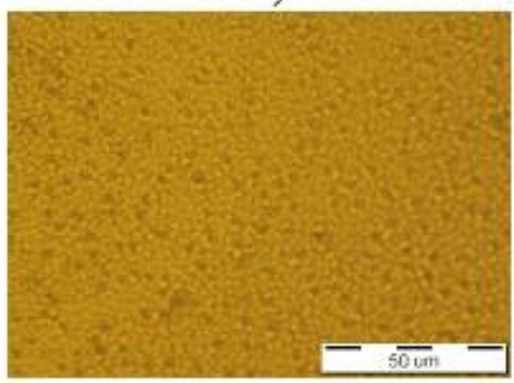

d)

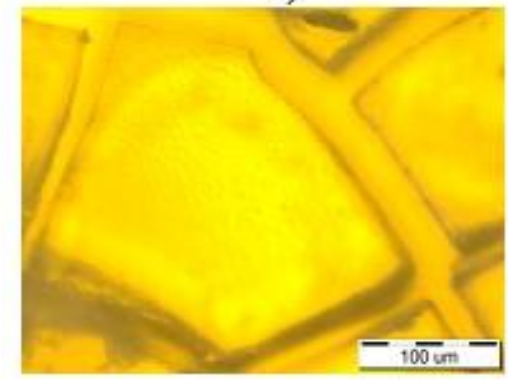

g)

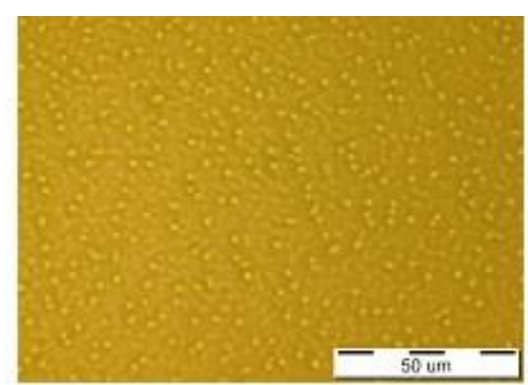

b)

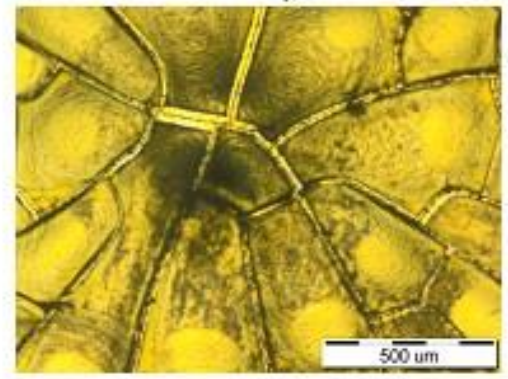

e)

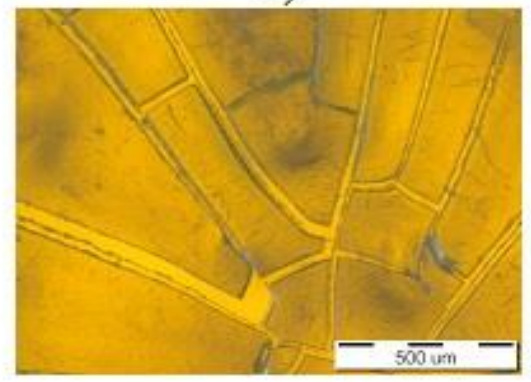

h)

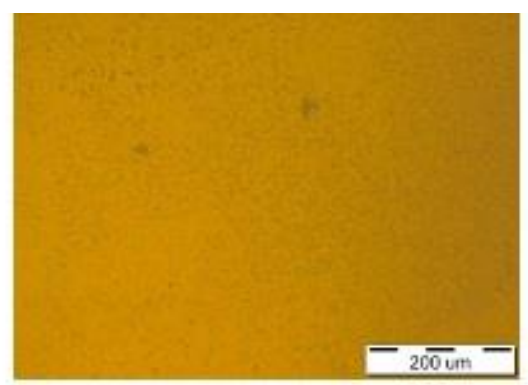

c)



f)



i)

Figure 4. The surface morphologies characterized by optical microscopy (OM) technique: a) and b) biosensor, c) and d) the biosensor with the addition of glucose, e), f) and g) the biosensor with the addition of glucose and serum, and $h$ ) and i) the biosensor with serum.

The absence of full coverage of the biosensor by ingredients already observed by the AFM technique is confirmed by the OM technique (Fig. 4a and b). It can be seen from Fig. 4a and 4b that the biosensor consists of small grains of spherical shape size uniformly distributed on Au electrode. The addition of glucose had not any effect on the appearance of the biosensor at the macro level, as shown in Fig. 4c and d. However, the remarkable change of surface morphology is observed by the addition of serum on the biosensor with the added glucose (Fig. 4e, $\mathrm{f}$ and $\mathrm{g}$ ). The appearance of the biosensor with added glucose and serum is spider's web-like without any similarity with previously observed surface morphologies of the biosensor. It is clear that this appearance of the surface area can be ascribed to crystallization of serum on the biosensor with glucose, because the similar surface morphology is observed when serum was added on the biosensor without glucose (Fig. 4h and i). It is necessary to note that the strong effect of serum on the surface morphology has been already observed in the case of sildenafil citrate [29], but different from those observed here. 
Anyway, it is necessary to note that similar reports dealing with the investigation of the effect of serum crystallization on morphology of the glucose biosensor by OM technique are not found in the literature. Among reports using OM technique for surface characterization of different forms of serum, it was found [40] that several crystal forms of recombinant and wild-type human serum albumin (HSA), such as a large rhombohedral-shaped crystals and trapezoidal blades, can be grown under suitable crystallization conditions. Investigating structural features of HSA, it was also shown [41] that globules or spherical particles can be formed by crystallization from $10 \%$ aqueous solution of HSA. A rod shaped micro crystals of copper complexes were formed with bovine serum albumin (BSA) using a superficial solution-based route [42]. On the other hand, a wire shaped structures of cadmium (II) complexes with BSA and HSA were formed indicating that optical microscopy is a useful tool to investigate the morphology of the formed nanostructures [43].

\section{CONCLUSION}

Electrochemical ability of presented biosensor exhibit a linear relationship between current $v s$. glucose from 1.5 to $8 \mathrm{mmol}^{-1}$ with the effective potential range from $-200 \mathrm{mV}$ to $400 \mathrm{mV}$ in a samples containing human serum. This potential range is in accordance with the $\mathrm{AuOH}$ formation on the gold surface enabling glucose oxidation. This indication that the Cys-GA-GOx film on Au surface is not compact and that there were some bare regions which remain catalytically active is supported by AFM and OM results. The construction and the nature of bonding of Au-Cys-GA-GOx biosensor layers is confirmed the FTIR study.

\section{ACKNOWLEDGEMENT}

We are thankful to the Ministry of Education, Science and Technological Development of the Republic of Serbia for the financial support (Grant No. ON172013 and ON172060).

\section{References}

1. M.N. Piero, G.M. Nzaro and J.M. Njagi, Asian J. Biomed. Pharm. Sci., 4 (2014) 1.

2. D.A. Antonetti, R. Klein and T.W. Gardner, N. Engl. J. Med., 366 (2012) 1227.

3. D.M. Nathan, S. Genuth, J. Lachin, P. Cleary, O. Crofford, M. Davis, L. Rand and C. Siebert. N. Engl. J. Med., 329 (1993) 977.

4. R.R. Holman, S.K. Paul, M.A. Bethel, D.R. Matthews and H.A. Neil, N. Engl. J. Med., 359 (2008) 1577.

5. G. Ghirlanda, M.A. Di Leo, S. Caputo, S. Cercone and A.V.Greco, Diabetes-Metab. Res., 13 (1997) 15.

6. H.W. van Dijk, F.D. Verbraak, P.H.B. Kok, M.K. Garvin, M. Sonka, K. Lee, J.H. DeVries, R.P.J. Michels, M.E.J. van Velthoven, R.O. Schlingemann and M.D. Abràmoff, Invest. Ophth. Vis. Sci., 51 (2010) 3404.

7. H.W. van Dijk, F.D. Verbraak, P.H.B. Kok, M.K. Garvin, M. Sonka, K. Lee, J.H. DeVries, R.P.J. Michels, M.E.J. van Velthoven, R.O. Schlingemann and M.D. Abràmoff, Invest. Ophth. Vis. Sci., 51 (2010) 3660.

8. J. Wang, Chem. Rev., 108 (2008) 814.

9. M. Cichomski, E. Tomaszewska, K. Kośla, W. Kozłowski, P.J. Kowalczyk and J. Grobelny, Mater. Charact., 62 (2011) 268.

10. N.J. Lang, B. Liu and J. Liu, J. Colloid Interface Sci., 428 (2014) 78. 
11. H. Zhang and N. Toshima, J. Colloid Interface Sci., 394 (2013) 166.

12. A. Ulman, Chem. Rev., 96 (1996) 1533.

13. L. Qingwen, G. Hong, W. Yiming, L. Guoan and M. Jie, Electroanal., 13 (2001) 1342.

14. S.K. Moccelini, S.C. Fernandes and I.C. Vieira, Sens. Actuators B, 133 (2008) 364.

15. J. Zhang, A. Demetriou, A. C. Welinder, T. Albrech, R. J. Nichols and J. Ulstrup, Chem. Phys., 319 (2005) 210.

16. P. Adlercreutz, Chem. Soc. Rev., 42 (2013) 6406.

17. Z. Yu, Y. Kou, Y. Dai, X. Wang, H. Wei and D. Xia, Electrocatalysis, 6 (2015) 341.

18. J. Liu, M. N. Paddon-Row and J.J. Gooding, Chem. Phys., 324 (2006) 226.

19. A. Salimi, R.G. Compton and R. Hallaj, Anal. Biochem., 333 (2004) 49.

20. R. Wilson and A. Turner, Biosens. Bioelectron., 7 (1992) 165.

21. A. Noorbakhsh, A. Salimi and E. Sharifi, Electroanal., 20 (2008) 1788.

22. T. Hoshi, N, Sagae, K. Daikuhara, K. Takahara and J. Anzai, Mat. Sci. Eng. C-Bio. S, 27 (2007) 890.

23. S. Sabury, S. Habib Kazemi and F. Sharif, Mat. Sci. Eng. C-Bio. S, 49 (2015) 297.

24. K. Tian, S. Alex, G. Siegel and A. Tiwari, Mat. Sci. Eng. C-Bio. S, 46 (2015) 548.

25. A. Rodrigues, M.V. Castegnaro, J. Arguello, M.C.M. Alves and J. Morais, Appl. Surf. Sci., 402 (2017) 136.

26. Y. Zhou, L. Wang, Z. Ye, M. Zhao, H. Cai and J. Huang, Appl. Surf. Sci., 285 (2013) 344.

27. J. Jung and S. Lim, Appl. Surf. Sci., 265 (2013) 24.

28. J. Lović, S. Stevanović, N.D. Nikolić, B. Andjelković, S. Petrović, D. Vuković, N. Prlainović, D. Mijin and M. Avramov Ivić, Int. J. Electrochem. Sci., 12 (2017) 5806.

29. J. Lović, N. Trišović, J. Antanasijević, N.D. Nikolić, S. Stevanović D. Mijin, D. Vuković, A. Mladenović, S. Petrović and M. Avramov Ivić, J. Electroanal. Chem., 782 (2016) 103.

30. K.M. Drljević-Djurić, V.D. Jović, U.Č. Lačnjevac, M.L. Avramov Ivić, S.D. Petrović, D.Ž. Mijin and S.B. Djordjević, Electrochim. Acta, 56 (2010) 47.

31. B. Andjelković, Lj. Vujisuć, I. Vučković, V. Tešević, V. Vajs and D. Godjevac, J. Pharmaceut. Biomed. 135 (2017) 217.

32. R. L. Brazg, L. J. Klaff and A.M. Sussman, J. Diabetes Sci. Technol., 10 (2016) 1414.

33. M. Pasta, F. La Mantia and Y. Cui, Electrochim. Acta, 55 (2010) 5561.

34. A. Pawlukojć, J. Leciejewicz, A.J. Ramirez-Cuesta and J. Nowicka-Scheibe, Spectrochim. Acta A, $61(2005) 2474$.

35. A. Barth, BBA-Bioenergetics, 1767 (2007) 1073.

36. A. Natalello, D. Ami, S. Brocca, M. Lotti and S.M. Doglia, Biochem. J., 385 (2005) 511.

37. J. Lambert, Introduction to Organic Spectroscopy, Mncmillan, Publ., (1987), New York, USA.

38. J.M. Bolivar, J. Rocha-Martin, C. Mateo, F. Cava, J. Berenguer, D. Vega, R. Fernandez-Lafuente and J.M. Guisan, J. Mol. Cat. B: Enzym, 58 (2009) 158.

39. D. Bezbradica, C. Mateo and J.M. Guisan, J. Mol. Catal. B: Enzym, 102 (2014) 218.

40. D.C. Carter, B. Chang, J.X. Ho, K. Keeling and Z. Krishnasami, Eur. J. Biochem., 226 (1994) 1049.

41. M.E. Buzoverya, I.V. Shishpor and Yu.P. Shcherbak, Tech. Phys., 63 (2018) 291.

42. M. Hazra, T. Dolai, A. Pandey, S.K. Dey and A. Patra, Journal of Saudi Chemical Society, 21 (2017) S240.

43. M. Hazra, T. Dolai, S. Giri, A. Patra and S.K. Dey, Journal of Saudi Chemical Society, 21 (2017) S445.

(C) 2018 The Authors. Published by ESG (www.electrochemsci.org). This article is an open access article distributed under the terms and conditions of the Creative Commons Attribution license (http://creativecommons.org/licenses/by/4.0/). 